\title{
Blind Search Methods for Binary Gamma-ray Pulsars
}

\author{
Lars Nieder* \\ Albert-Einstein-Institut, Max-Planck-Institut für Gravitationsphysik, D-30167 Hannover, \\ Germany \\ Leibniz Universität Hannover, D-30167 Hannover, Germany \\ E-mail: lars.nieder@aei.mpg.de
}

Colin J. Clark ${ }^{\dagger}$

Albert-Einstein-Institut, Max-Planck-Institut für Gravitationsphysik, D-30167 Hannover,

Germany

Leibniz Universität Hannover, D-30167 Hannover, Germany

Jodrell Bank Centre for Astrophysics, School of Physics and Astronomy, The University of Manchester, Manchester M13 9PL, UK

\section{on behalf of the Fermi-LAT Collaboration}

Gamma-ray observations by the Fermi Large Area Telescope have been used very successfully in the last 9 years to detect more than 200 gamma-ray pulsars. Sixty of these have been found by directly searching for pulsations in the gamma-ray data, but only one binary MSP has been found this way. Pulsars in binaries are often difficult to detect in radio data because of large eclipses, and some binary MSPs may even be radio quiet. For those, a gamma-ray blind search might be the only possibility for detection. While searches for isolated pulsars up to kilohertz frequencies are already computationally very challenging, blind searches for binary gamma-ray pulsars are simply infeasible without further knowledge of their orbital parameters. We present methods with which we can conduct searches for candidate binary gamma-ray pulsars for which orbital constraints are known from optical observations of a likely companion star. We also highlight some example sources where these methods have been used. Additionally, some redback MSPs can be more easily timed in gamma rays than with radio observations.

7th Fermi Symposium 2017

15-20 October 2017

Garmisch-Partenkirchen, Germany

\footnotetext{
* Speaker.

${ }^{\dagger}$ C.J.C. acknowledges support from the ERC under the European Union's Horizon 2020 research and innovation programme (grant agreement No. 715051; Spiders).

${ }_{\ddagger}^{\ddagger}$ The Fermi-LAT Collaboration acknowledges support for LAT development, operation and data analysis from NASA and DOE (United States), CEA/Irfu and IN2P3/CNRS (France), ASI and INFN (Italy), MEXT, KEK, and JAXA (Japan), and the K.A. Wallenberg Foundation, the Swedish Research Council and the National Space Board (Sweden). Science analysis support in the operations phase from INAF (Italy) and CNES (France) is also gratefully acknowledged. This work performed in part under DOE Contract DE-AC02-76SF00515.
} 